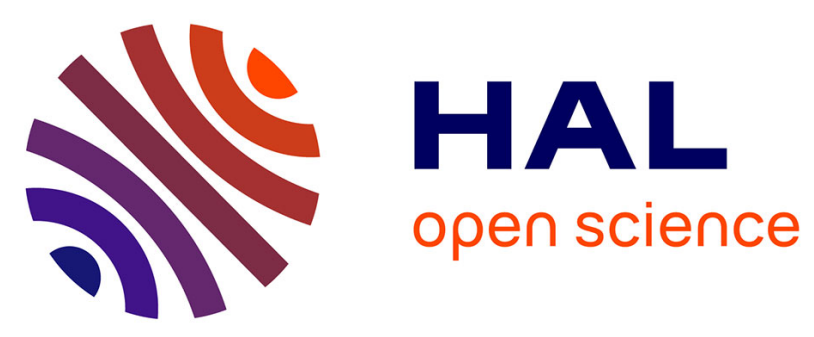

\title{
Jean Potocki méritait mieux
}

Daniel Beauvois

\section{To cite this version:}

Daniel Beauvois. Jean Potocki méritait mieux. Dix-Huitième Siècle, 1990, Voyager, explorer, 22, pp.441-449. halshs-01086545

\section{HAL Id: halshs-01086545 \\ https://shs.hal.science/halshs-01086545}

Submitted on 24 Nov 2014

HAL is a multi-disciplinary open access archive for the deposit and dissemination of scientific research documents, whether they are published or not. The documents may come from teaching and research institutions in France or abroad, or from public or private research centers.
L'archive ouverte pluridisciplinaire HAL, est destinée au dépôt et à la diffusion de documents scientifiques de niveau recherche, publiés ou non, émanant des établissements d'enseignement et de recherche français ou étrangers, des laboratoires publics ou privés. 


\section{JEAN POTOCKI MÉRITAIT MIEUX}

Le chœur des critiques de la grande presse a salué la sortie chez José Corti de la " première édition intégrale » du Manuscrit trouvé à Saragosse comme un événement majeur de la vie littéraire française et cela peut sembler juste.

Le public français découvre, pour la première fois, dans toute l'ampleur de ses soixante-six "journées", un texte merveilleux et foisonnant, témoignage capital des Lumières européennes.

Quel dommage que la mise au point d'un ouvrage aussi fondamental n'ait pas été faite dans le respect des règles scientifiques et déontologiques d'usage! Dans l'édition Corti, présentée comme intégrale, quatorze journées (sur soixante-six) ne sont pas conformes à l'original. Cette édition ne rend surtout aucun compte de plusieurs variantes capitales alors qu'elle s'affirme "basée sur la totalité des sources accessibles".

Rappelons seulement que Jean Potocki n'avait fait imprimer, avant sa mort en 1815, que les premières journées de son roman, parues à Saint-Pétersbourg et Paris, laissant l'ensemble du

Manuscrit trouvé à Saragosse en copies manuscrites éparses chez des proches. Après quelques plagiats partiels et les scandales qui s'ensuivirent en France au début des années quarante du $19^{\mathrm{e}}$ siècle (affaires Nodier et Courchamps), un traducteur polonais, Edmond Chojecki, dit Charles Edmond, donna à Leipzig, en 1847, une traduction "complète" en polonais. Les copies françaises de l'original semblèrent alors tomber dans l'oubli et personne ne s'en préoccupa plus. Lorsque Roger Caillois remit Potocki en honneur, en 1958, il ne publia, chez Gallimard, que les treize journées déjà imprimées du vivant de l'auteur, mais l'intérêt était relancé et la postface d'une nouvelle édition polonaise due à L. Kukulski, en 1965, donnait déjà les cotes des quatrième et cinquième "décamérons" originaux déposés dans des archives polonaises

1. Manuscrit trouvé à Saragosse, de Jean Potocki. Première édition intégrale tablie par René Radrizzani (Paris, José Corti, 1989), xx + 683 p. 
Un colloque, organisé en avril 1972 par le Centre de Civilisation Française de l'Université de Varsovie, fut consacré à l'œuvre de Jean Potocki (actes dans les Cahiers de Varsovie, $\mathrm{n}^{\circ} 3$, rééd. 1981) : et le monde littéraire sut, dès lors, que $\mathbf{M}^{\mathrm{me}}$ Marie Évelyne Zóltowska, auteur d'une thèse sur Potocki ${ }^{2}$, préparait l'édition définitive du texte français, avec R. Caillois d'abord, qui souhaitait retraduire du polonais les fragments "disparus", puis seule, après la mort de celui-ci. Au prix d'enquêtes longues et minutieuses, cette chercheuse américano-polonaise finit par identifier 17 manuscrits partiels du roman de Jean Potocki et signa avec Gallimard un contrat pour une publication dans la Pléiade. Sauf peut-être encore quelques pages manquantes, M. E. Zóltowska est la seule personne en possession de la totalite du Manuscrit trouvé à Saragosse en français, ainsi que d'importantes variantes.

Il faut avouer que pendant toutes ces années l'impatience grandissait de voir enfin l'œuvre dévoilée dans son intégralité Plus d'un chercheur maudissait les lenteurs de notre consœur que la complexité des variantes (et le poids de problèmes familiaux) freinait dans son travail ; mais, malgré l'impression générale que les choses auraient pu aller plus vite, chacun en attendait la révélation, respectueux des recherches et du travail de notre collègue : D. Triaire, en publiant, en 1985, l'Inventaire de l'Euvre de J. Potocki chez Champion, s'interdit de toucher le Manuscrit trouvé à Saragosse.

Les éditions José Corti, malheureusement, passèrent outre aux réserves qu'appelait une telle situation. La fièvre monta de quelques degrés en 1988, lorsqu'un important fragment du Manuscrit fut découvert par hasard aux archives municipales de Pontarlier (voir J. R. Dahan, «Une copie inconnue du Manuscrit trouvé à Saragosse ", R.H.L.F. vol. 89, n 2 (mars avril 1989) p. 260-266). Cette copie permettait d'augmenter l'édition Caillois d'un bon tiers et, dès lors, la tentation devenait encore plus forte d'utiliser la dizaine de cotes d'archives connues d'assembler rapidement ces textes et de les livrer au public avant Gallimard.

Cette recherche de ce qui ressemble à un "coup" éditorial. venant d'une maison qui nous avait habitués à plus de rigueu

2. M. E. Zóltowska, Un précurseur de la littérature fantastique : Jean Potocki. sa vie et son "Manuscrit trouvé à Saragosse", Yale University, 1973, VII 676 p. (Ann Arbor, University Micro-films, 1973) [disponible à la Bibliothèque nationale depuis 1980 , cote : microfilm m.15025 intellectuelle, porte un préjudice sensible (même s'il n'est que temporaire) à la connaissance des Lettres.

Il a, en effet, fallu recourir, pour boucler cette édition, à la retraduction de quatorze journées. Si l'esprit, le contenu narratif, sont rendus, on conviendra que la lettre, le style inimitable de Potocki sont perdus ${ }^{3}$. Or les responsables de Corti ne pouvaient ignorer l'existence des 17 manuscrits de M. E. Zóltowska : je leur en avais moi-même parlé lorsqu'ils m'interrogèrent par téléphone sur l'état de la question (sans me parler de leur intention) et surtout J.-R. Dahan les mentionnait dans l'article cité plus haut.

Les chercheurs désireux de connaître la teneur des chapitres inédits en français pouvaient, depuis plusieurs années déjà, consulter la traduction polono-française donnée en annexe à la thèse dactylographiée de troisième cycle soutenue par Wanda Rapacka à Strasbourg. C'est donc d'abord vers elle que Corti se tourna pour boucher les trous de son édition. Elle refusa, naturellement, au nom de l'honnêteté intellectuelle, précisant, elle aussi, que les trouvailles de M. E. Zóltowska rendaient sa traduction obsolète.

C'est ici qu'intervint René Radrizzani, professeur d'allemand au Gymnase de la Cité à Lausanne, qui se proposa pour composer le volume désiré. L'édition Corti ne donnant aucun détail quant à la base des journées retraduites, je m'enquis de la manière dont celui-ci avait travaillé. M. Fillaudeau (directeur des éditions J. Corti) m'expliqua d'abord que l'universitaire suisse avait spécialement appris le polonais, mais l'intéressé me déclara, au cours d'un entretien, qu'il ne connaissait pas cette langue et qu'il avai re-retraduit ses textes manquants d'après une édition en allemand parue chez Haffmans Verlag à Zurich en 1984 (d'abord parue à Berlin chez Aufbau Verlag en 1981 d'après l'édition polonaise de Kukulski). Donc 14 chapitres (140 pages) sont traduits d'un texte allemand (lui-même traduit du polonais), sans le dire nulle part et en sachant que $\mathrm{M}^{\mathrm{me}} \mathrm{M}$. E. Zóltowska en possède l'original en français ! Et cela n'empêche pas d'annoncer sur la page de couverture qu'il s'agit de la « première édition intégrale » d'un chef-d'œuvre de la littérature en français...

Le plus déplorable en cette affaire est évidemment le manque de coopération entre deux chercheurs qui ont travaillé séparé-

3. Sur les insuffisances de la traduction, voir l'article sévère de Nicole Taillade dans Littératures, ${ }^{\circ} 21$ (automne 1989), p. 181-184. 
ment, le moins bien informé ayant su être le plus rapide. Comment R. Radrizzani ignorerait-il les découvertes de M. E. Zóltowska et comment expliquer cette note 9 de la page 675 disant que "toutes les copies semblent perdues" ? Pourquoi n'avoir pas que "toutes les copies semblent de base de verifié la légende de la Chojecki ? Un chercheur ne peut ignorer les publications antérieures et encore moins répéter, comme il est fréquent ici, les rieure elles. La bibliographie (p. 676) es très insuffisante. Il n'est pas non plus permis de s'arroger la paternité de découvertes faites par d'autres, or les notes de l'introduction et les remarques sur l'établissement du texte n'indiquent jamais d'où ces données sur la situation des sources sont puisées, laissant croire à une originalité des recherches qui est toute fictive. Par exemple R. Radrizzani reproduit, pour les frag ments qu'il publie, des cotes d'archives qu'il a trouvées dans des ouvrages édités (la thèse de $\mathrm{M}^{\mathrm{me}}$ Zóltowska - mentionnée nulle part ${ }^{4}$ - et les Actes du Colloque Potocki).

Qu'importent ces querelles de puristes, diront les amateurs, les journées re-retraduites sont les dernières et de beaucoup les plus courtes du roman, si bien que le volume de texte nouveau et original mis à la disposition du public est quand même considérable. Soit. Mais Potocki méritait moins de légèreté.

Précision: Je profite de l'occasion pour apporter un complément d'information à l'article de Jacques-Rémi Dahan cité cidessus (R.H.L.F. 1989, mars-avril) et relatif au manuscrit de Pontarlier contenant les vingt premières journées. J.-R. Dahan suppose que ces deux décamérons ont été remis directement à Auguste de Saint-Aignan par Potocki "bien que, dit-il, nous n'ayons pas découvert de preuve formelle de leur rencontre". Je n'ayons pas découver que j'ai établi la réalité et l'importance des me permets d'indiquer que j'ai établi la réalité et l'importance des rapports entre ces deux hommes dans mon article « Le "Systèm asiatique" de Jean Potocki ou le rêve oriental dans les empire d'Alique de Jean d'Alexandre I et de Napoléon, 1806-1808 ", Cahiers du Monde Russe et Soviétique XX, (3-4) (juillet-décembre 1979), p. 469 471 , ainsi que dans le tome 2 de mon édition des Voyages de J. Potocki (Paris, Fayard, 1980), p. 24-25. La présence de ce texte à Pontarlier s'explique ainsi tout naturellement et la

4. Voir un article de protestation (en polonais) de $\mathbf{M}^{\mathrm{me}} \mathrm{M}$. E. Zóltowska, au itre éloquent : "Le manuscrit trouvé dans une thèse", Kultura (Paris), novetnbe 1989 , p. $116-123$ confiance de Jean Potocki pour Auguste de Saint-Aignan trouve là une belle illustration.

DANIEL BEAUVOIS

Université Charles de Gaulle (Lille III)

Nous avons communiqué l'article qu'on vient de lire aux Éditions Corti en leur proposant de répondre à notre collaborateur. Nous publions donc cette réponse qui comporte deux textes (R.D.).

Monsieur Daniel Beauvois par malveillance et non méconnaissance nous accuse notamment d'avoir cherché dans la publication du Manuscrit trouvé à Saragosse un "coup éditorial" sans respecter les règles scientifiques et déontologiques d'usage. Nous demandons en conséquence que cette lettre soit insérée comme droit de réponse à ce "coup bas".

Cela est d'autant plus extraordinaire qu'une lettre du 16 août 1989 de M. Daniel Beauvois ne laissait pas présager une telle hargne : "Votre belle édition du Manuscrit trouvé à Saragosse provoque un intérêt et une curiosité bien légitimes quand il s'agit d'un texte si important. »C'est à la suite de cette lettre que M. Beauvois rencontra René Radrizzani.

Un peu d'histoire d'abord - nous pouvons fournir à qui le voudra la copie des lettres l'attestant.

Durant l'été 1987 (et non en 1988) Jacques-Rémi Dahan me contacta après avoir découvert dans les archives municipales de Pontarlier un important fragment du Manuscrit trouvé à Saragosse. Il se proposait ainsi d'améliorer l'édition de Roger Caillois en utilisant éventuellement le travail de $\mathrm{M}^{\mathrm{me}}$ Rapacka avec qui nous eûmes une réunion à Paris.

J'écrivis alors à $\mathrm{M}^{\mathrm{me}}$ Zóltowska pour lui faire part de la situation dans laquelle je me trouvais. La réponse de $\mathrm{M}^{\text {me }}$ Zóltowska me conduisit à abandonner le projet d'édition partielle peu satisfaisante, ce dont j'informai $\mathbf{M}^{\text {me }}$ Rapacka et Jacques-Rémi Dahan.

Jacques-Rémi Dahan décida alors de faire part de sa découverte dès décembre 1987. 
En octobre 1988, je reçus un appel téléphonique de René Radrizzani qui ne connaissait pas la découverte de Jacques-Rémi Dahan, ni la "chasse gardée" de $\mathbf{M}^{\text {me }}$ Zóltowska. Enthousiasmé par le Manuscrit trouvé à Saragosse qu'il avait découvert dans sa traduction intégrale allemande, il avait fait venir les manuscrits indiqués dans cette édition et l'édition polonaise ; grâce à l'aide des bibliothèques, il disposait d'un ensemble de manuscrits et se mit à travailler en solitaire sur une version intégrale française entouré d'une équipe d'amis.

Nous pouvons prouver qu'aucun lien n'existait alors entre les recherches de Jacques-Rémi Dahan, celles de $\mathbf{M}^{\mathrm{me}}$ Zóltowska et celles de René Radrizzani qui, lui, peut prouver que les travaux de $\mathrm{M}^{\mathrm{me}}$ Zóltowska ne lui furent communiqués (correspondance avec les bibliothèques) qu'alors qu'il corrigeait les épreuves de la première édition.

Au nom de quoi, un éditeur digne de ce nom et à qui le hasard faisait ces clins d'œil répétés aurait-il pu repousser un tel texte?

Que M. Beauvois dise que notre édition n'est pas l'édition critique qu'il attendait est son droit le plus strict, de même qu'il fasse grief à notre édition de n'avoir pas retenu "des variantes capitales". Encore serait-il plus sérieux d'assortir ces affirmations d'exemples.

Une nouvelle édition qui tient compte des nouveaux fragments de manuscrits découverts et des remarques amicales de certains lecteurs vient d'ailleurs de paraître en janvier 1990. Elle atteste notre souci de donner à lire au public une version encore améliorée. Elle montre en outre que les nouveaux fragments découverts grâce à Marek Potocki, descendant de Jean Potocki, corroborent les options prises par René Radrizzani dans sa première édition.

Que M. Beauvois pense que le style de Potocki est perdu dans les quatorze journées traduites est là encore son droit de critique. Il est le premier à faire cette remarque, et ce qui précède comme ce qui suit nous permet de douter de son objectivité.

Ce qui est inacceptable, c'est que M. Beauvois ne nous ait pas parlé de ces accusations et qu'il réserve ces calomnies auxquelles nous pouvions répondre - à un article.

$\mathrm{Si}$ nous trempions notre plume dans le même vinaigre que M. Beauvois nous pourrions nous étonner qu'une revue sérieuse comme Dix-Huitième siècle ait accueilli un tel "coup bas" accepte d'accueillir un texte où la critique littéraire cède le pas à la diffamation.
M. Beauvois digère-t-il mal le fait qu'un non-spécialiste marche sur ses brisées, réussisse là où d'autres ont sinon échoué du moins buté même temporairement ? Une telle mauvaise foi est étonnante venant d'un universitaire. Quelle mouche, quels intérêts ont donc piqué $\mathrm{M}$. Beauvois ?

ÉDITIONS JOSÉ CORTI

A la demande de mon éditeur, je vous écris uniquement pour préciser deux points qui offrent de l'intérêt pour les lecteurs

1) Les passages pour lesquels les sources françaises faisaient défaut ont, comme je le signale aux p. 670,671 et 674 de mon édition, été retraduits avec l'aide de $\mathrm{M}^{\mathrm{me}}$ Yolanta SkupinskaJankowska à partir de la version polonaise de Chojecki, qui a par ailleurs été collationnée intégralement avec toutes les autres sources. S'il est vrai que j'ai consulté en outre deux éditions allemandes (ce que M. B. ne sait que parce que je le lui ai dit moi-même, en fournissant à sa demande la référence et la description de ces éditions), c'est cependant bien Chojecki qui constitue la référence de base.

2) Je ne pense pas qu'il manque, sur l'ensemble de près de 1000 variantes qu'offre l'édition, des « variantes capitales ». Je serais toutefois reconnaissant à toute personne qui me signalerait une erreur ou une omission.

RENÉ RADRIZZANI

$$
\text { ** }
$$

La réponse du directeur des Éditions Corti appelle une mise au point. Contrairement à ce qu'il écrit, son texte n'entre pas dans le cadre du "droit de réponse » prévu par la loi. C'est à titre de courtoisie et eu égard à l'estime dans laquelle nos lecteurs tiennent sa maison d'édition (qui fêtait son cinquantenaire en 1988) que je lui ai envoyé l'article de Daniel Beauvois en lui proposant d'y répondre et en m'engageant à publier sa réponse à la suite. Nul ne m'obligeait à le faire. J'ajoute que les termes utilisés à l'endroit de notre collaborateur ne me paraissent pas compatibles avec l'exercice du "droit de réponse » tel qu'il est réglementé.

Cela dit, puisque notre revue est interpellée, je tiens à préciser que c'est à ma demande, suite à la proposition de notre Conseil d'Administration, que D. Beauvois a rendu compte de l'édition de R. Radrizzani. Démarche toute naturelle. Réputé pour sa haute 
compétence dans le domaine de la littérature polonaise du $18^{e}$ siècle et, particulièrement, pour ce qui touche à Potocki, D. Beauvois l'Université de Varsovie, qui avait organisé, avec le concours de Roger Caillois, le colloque de 1972 sur Potocki et le Manuscrit trouvé à Saragosse. En recevant son article, nous n'avons pas eu droit à la critique. Puisque les Editions Corti reconnaissent ce droit, pourquoi notre revue ne l'aurait-elle pas respecté ?

A la question "quelle mouche, quels intérêts ont donc piqué $M$. Beauvois ?", la réponse devrait aller de soi pour tout lecteur attentif. Si ce n'était pas le cas, je dirais, au nom de tous ceux qui le connaissent, que D. Beauvois n'a, ici, pour souci et pour intérêts que ceux de la recherche de la vérité et d'un meilleur et plus large accès aux grandes œuvres littéraires. Les améliorations que comportent la deuxième édition (qui a paru quand nous mettions sous presse) ne vont-elles pas dans le sens de ce que souhaitait notre collaborateur? et ne justifient-elles pas sa critique de la première édition? Il n'en demeure pas moins que subsistent encore, dans cette nouvelle édition, une centaine de pages traduites de l'allemand.

L'éditeur Corti confirme aussi la sévérité du jugement porté par $D$. Beauvois lorsqu'il révèle que R. Radrizzani n'a eu connaissance des travaux de $M^{\text {me }}$ Zóltowska qu'au moment où il corrigeait les épreuves de son édition (soit vers le printemps 1989). Que penserait-on de l'auteur d'une édition critique de La Religieuse qui attendrait d'en corriger les épreuves pour consulter les travaux de H. Dieckmann? Et comment comprendre que le même éditeur qui se met en contact avec $M^{\text {me }}$ Zóltowska en 1987, dès qu'un premier projet de publication du Manuscrit s'est formé, laisse si longtemps M. Radrizzani dans l'ignorance? Tout en s'interrogeant sur l'ampleur des corrections et des additions qui ont éte faites sur les épreuves, on peut donc se demander si cette édition n'a pas été réalisée un peu hâtivement.

On voit ainsi, à la lecture de l'article de D. Beauvois et de la réponse des Éditions Corti, se dessiner les logiques différentes du métier de chercheur et du métier d'éditeur. Ces métiers se complètent. Éditeurs et chercheurs ont besoin les uns des autres, et les services rendus à la recherche littéraire par les Editions Cort sont assez notoires pour fournir une belle illustration de cett complémentarité. Mais les impératifs du chercheur motivé par son désir de faire progresser le savoir, de vérifier ses découvertes et ses conjectures par des enquêtes souvent recommencées, et prenant le temps qu'il lui faut, ne sont pas ceux de l'éditeur soucieux, légitimement, de faire vivre sa maison, de satisfaire (ou provoquer) l'attente des lecteurs et, pour cela de répondre aux "clins d'ceil" $d u$ " hasard —- en d'autres termes, saisir les chances offertes sur le marché de l'édition. Que les Éditions Corti continuent de tenir une place exemplaire dans ce marché - devenu aujourd'hui plus difficile et plus périlleux -, c'est le vœu de tous les dix-huitiémistes, mais qu'elles comprennent qu'il est malhabile de répondre à la critique par l'insulte.

ROLAND DESNÉ

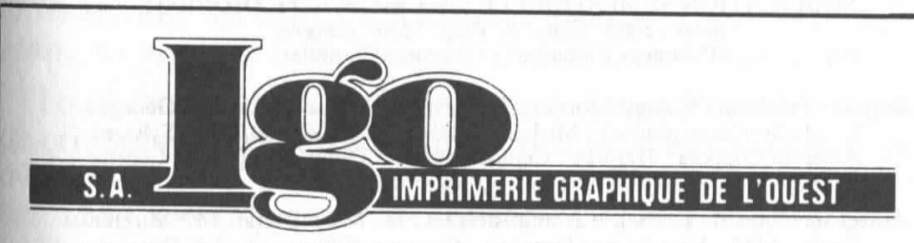

\section{LE SPECIALISTE DU LIVRE ET DU CATALOGUE}

\section{LANCE UN ATELIER DE PETITS TIRAGES}

B.P. 16

Chemin des Amours Le Poiré-sur-Vie

85170 BELLEVILLE-SUR-VIE

Tél : $51.06 .46 .00-51.06 .45 .73$ 